\title{
Sensitivity of the upgraded T2K Near Detector to constrain neutrino and antineutrino interactions with no mesons in the final state by exploiting nucleon-lepton correlations
}

\author{
S. Dolan $\odot,{ }^{1, *}$ V. Q. Nguyen $\odot,{ }^{2, \dagger}$ A. Blanchet $\odot,{ }^{2}$ S. Bolognesi, ${ }^{3}$ M. Buizza Avanzini $\odot,{ }^{4}$ J. Chakrani, ${ }^{4}$ A. Ershova $\odot,{ }^{3}$ \\ C. Giganti, ${ }^{2}$ Y. Kudenko $\odot,{ }^{5,6,7}$ M. Lamoureux $\odot,{ }^{8}$ A. Letourneau $\odot,{ }^{3}$ M. Martini $\odot,{ }^{2,9}$ C. McGrew, ${ }^{10}$ L. Munteanu $\odot,{ }^{1,3}$ \\ B. Popov $\oplus^{2}$ D. Sgalaberna ${ }^{11}{ }^{1}$ S. Suvorov, ${ }^{2,5}$ and X. Y. Zhao $\circledast^{11}$ \\ ${ }^{1}$ CERN European Organization for Nuclear Research, CH-1211 Geneva 23, Switzerland \\ ${ }^{2}$ LPNHE, Sorbonne Université, CNRS/IN2P3, 75005 Paris, France \\ ${ }^{3} I R F U, C E A$, Université Paris-Saclay, F-91191 Gif-sur-Yvette, France \\ ${ }^{4}$ Laboratoire Leprince-Ringuet (LLR), CNRS, Ecole polytechnique, Institut Polytechnique de Paris, \\ 91120 Palaiseau, France \\ ${ }^{5}$ Institute for Nuclear Research of the Russian Academy of Sciences, 117312 Moscow, Russia \\ ${ }^{6}$ Moscow Institute of Physics and Technology (MIPT), 141700 Moscow, Russia \\ ${ }^{7}$ National Research Nuclear University MEPhI, 115409 Moscow, Russia \\ ${ }^{8}$ INFN Sezione di Padova, I-35131 Padova, Italy \\ ${ }^{9}$ IPSA-DRII, 94200 Ivry-sur-Seine, France \\ ${ }^{10}$ State University of New York at Stony Brook, Department of Physics and Astronomy, \\ Stony Brook, New York 11794, USA \\ ${ }^{11}$ ETH Zurich, Institute for Particle Physics and Astrophysics, CH-8092 Zurich, Switzerland
}

(Received 14 September 2021; accepted 27 January 2022; published 28 February 2022)

\begin{abstract}
The most challenging and impactful uncertainties that future accelerator-based measurements of neutrino oscillations must overcome stem from our limited ability to model few-GeV neutrino-nucleus interactions. In particular, it is crucial to better understand the nuclear effects which can alter the final state topology and kinematics of neutrino interactions, inducing possible biases in neutrino energy reconstruction. The upgraded ND280 near detector of the T2K experiment will directly confront neutrino interaction uncertainties using a new suite of detectors with full polar angle acceptance, improved spatial resolutions, neutron detection capabilities and reduced tracking thresholds. In this manuscript we explore the physics sensitivity that can be expected from the upgraded detector, specifically focusing on the additional sensitivity to nuclear effects and how they can be constrained with future measurements of kinematic variables constructed using both outgoing lepton and nucleon kinematics.
\end{abstract}

DOI: 10.1103/PhysRevD.105.032010

\section{INTRODUCTION}

Neutrino oscillations are measured at accelerator-based experiments by inferring the rate of interactions of a particular neutrino flavor, as a function of neutrino energy, at a detector placed some distance from the neutrino production point (the "far detector"). However, in order to meaningfully interpret far detector data in terms of neutrino oscillation probabilities, it is essential to well understand the unoscillated incoming neutrino flux, the

\footnotetext{
Stephen.Joseph.Dolan@ cern.ch

quocviet.nguyen@lpnhe.in2p3.fr
}

Published by the American Physical Society under the terms of the Creative Commons Attribution 4.0 International license. Further distribution of this work must maintain attribution to the author(s) and the published article's title, journal citation, and DOI. Funded by SCOAP. detector response and the relevant neutrino interaction cross sections. It is also crucial to be able to estimate the bias and smearing of neutrino energy estimators due to nucleareffects in neutrino interactions [1]. To constrain systematic uncertainties in oscillation measurements, neutrino-beam experiments therefore usually employ an additional detector placed close to the beam production point (the "near detector"), before any oscillation is expected to have occurred. Measurements at the near detector are then used to constrain predictions at the far detector.

T2K [2] and Hyper-Kamiokande ("Hyper-K") [3] are long-baseline experiments which measure neutrino oscillations over a distance of $295 \mathrm{~km}$ using intense muon neutrino and antineutrino beams with a neutrino energy peak at $\sim 600 \mathrm{MeV}$. The upgraded ND280 detector ("ND280 Upgrade") [4] considered in this article is an improvement of the existing near detector of the T2K experiment ("ND280"), which will also serve as a key 


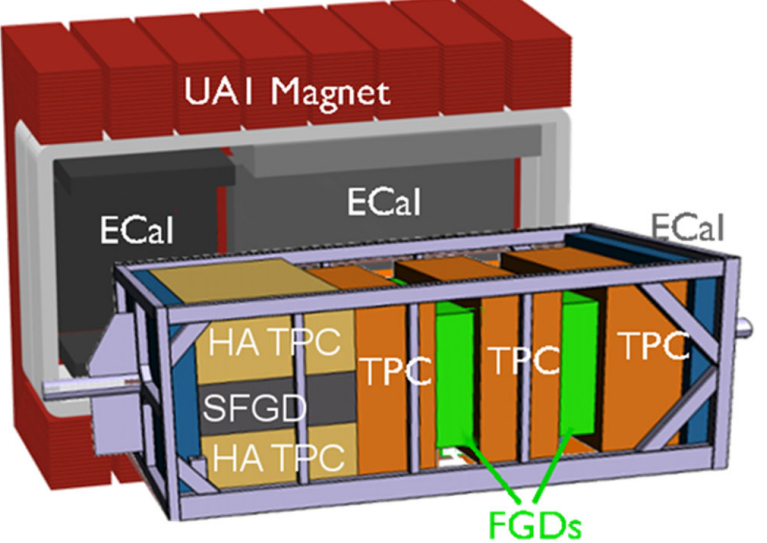

FIG. 1. An exploded view of the ND280 Upgrade detector. Neutrinos enter from the left of the image. Figure adapted from Refs. $[4,10]$.

component of the upcoming Hyper-K experiment's near detectors. The complete ND280 Upgrade detector consists of several subdetectors encased in a magnet providing a uniform $0.2 \mathrm{~T}$ field, as shown in Fig. 1. The ND280 upgrade will retain the downstream sub-detectors of the current ND280, comprising a central tracking region consisting of "finegrained" scintillator detectors (FGDs) [5] to act as targets for neutrino interactions, and low-density gaseous time projection chambers (TPCs) [6] to precisely track emitted charged particles, surrounded by an electromagnetic calorimeter (ECal). The new components of in the upgrade will replace the current upstream ND280 subdetectors with two horizontal "high angle" TPCs (HA-TPC) and the horizontal "Super-FGD" [4]. The former are improved versions of the existing TPCs which will significantly extend the detector's angular acceptance and resolution [7], while the latter is a new type of active target for neutrino interactions, made of more than 2 million $1 \mathrm{~cm}^{3}$ optically isolated scintillating cubes connected with readout fibres in three orthogonal directions [8]. Six time-of-flight planes will be installed around the HA-TPCs and the Super-FGD to verify whether particles crossing the Super-FGD are leaving or entering the active volume [9] and to provide complementary information for particle identification.

ND280 Upgrade is especially well adapted to characterize the aspects of neutrino interactions that are responsible for some of the most impactful and challenging sources of systematic uncertainties in $\mathrm{T} 2 \mathrm{~K}$ measurements. In particular, the $3 \mathrm{D}$ readout and fine segmentation of the Super-FGD allows for an excellent hadron reconstruction with very low thresholds in a hydrocarbon material. For example, the Super-FGD proton tracking momentum threshold is around $300 \mathrm{MeV} / \mathrm{c}$, compared to the current FGD's $450 \mathrm{MeV} / \mathrm{c}$ threshold [4]. Such lower threshold tracking allows a significantly improved characterization of the nuclear effects that can bias neutrino energy reconstruction [1] and which were responsible for some of the largest uncertainties in
T2K's latest neutrino oscillation analysis $[11,12]$. In addition, the Super-FGD's neutron detection capabilities [4] both extend its sensitivity to antineutrino interactions and allow a potential isolation of an almost nuclear-effect free sample of neutrino interactions on hydrogen [13]. Since neutron detection does not rely on particle tracking, but instead on the identification of neutron-nucleus secondary interactions displaced from the neutrino interaction vertex, and there exists an abundance of hydrogen within the Super-FGD to facilitate detection of low energy elastic scatters, there is no important hard neutron detection threshold.

The goal of this paper is to provide a quantitative estimate of ND280 Upgrade's sensitivity to the most important sources of systematic uncertainty in T2K and Hyper-K oscillation analyses, specifically through its ability to accurately reconstruct nucleons alongside the charged lepton in charged-current interactions with no mesons in the final state (the primary interaction topology for T2K and Hyper-K). These sensitivities are presented as a function of accumulated statistics, covering both the T2K and Hyper-K eras and given by the number of protons impinging on the target (POT) in the neutrino beamline. For reference, T2K's latest analysis used 1.49(1.64) $\times 10^{21}$ POT of neutrino (antineutrino) data [12] and will have an ultimate total exposure up to $10 \times 10^{21}$ POT, while the Hyper-K design report [3] considered a total of $27 \times 10^{21}$ POT (in a 1:3 neutrino over antineutrino ratio), corresponding to 10 years of data taking. The analysis method is presented in Sec. II, where the simulation and the reconstruction are described, followed by a discussion of the strategy to extract meaningful sensitivities. The resultant sensitivities are then discussed in Sec. III before conclusions are drawn in Sec. IV.

\section{METHODOLOGY}

\section{A. Simulation}

While the full simulation of the ND280 Upgrade is in active development within the $\mathrm{T} 2 \mathrm{~K}$ collaboration, the fundamental performances of the proposed detector technology have been extensively studied in various test-beams at CERN [7,14], LANL and DESY [15]. These data have allowed development of a preliminary Super-FGD detector simulation and reconstruction framework. From this early simulation, the main detector effects (smearing and resolution) have been parametrized and applied to a sample of neutrino interactions generated with the NEUT simulation $[16,17]$ on a hydrocarbon scintillator $(\mathrm{CH})$ target using the T2K neutrino and antineutrino flux predictions $[18,19]$.

Within the reconstruction framework, charge deposits in the Super-FGD are identified and processed to form tracks. A small region very close to the interaction vertex is willingly neglected in order to negate the impact of energy deposition from other interaction products in the vicinity of the target nucleus. For contained tracks, the charge deposits along most of the track's length are considered to identify the particle energy loss over the whole track range, 
permitting both momentum reconstruction and particle identification (PID). Tracks with an ambiguous PID and those which undergo apparent secondary interactions (i.e., from an observed deflection of peak in the charge deposits) are rejected. ${ }^{1}$ It should be noted that the nonuniformity of energy deposits due to the position of particles within a scintillator cube is not yet modeled (which could worsen the relative momentum resolution for short tracks by $\sim 10 \%$ ), but it has been ensured that such changes in resolution would not significantly affect the results presented here. Tracks which are not fully contained and enter a TPC are split into two parts: the Super-FGD segment is reconstructed as described above and the TPC segment undergoes a parameterized reconstruction based on known performances of the current T2K TPC [6]. The improved performance expected from the HA-TPCs is not yet taken into account (meaning the detector performance for the TPC segments of high angle tracks is expected to be slightly underestimated).

The input NEUT (version 5.4.0) simulation uses the spectral function model [20] for charged-current quasielastic (CCQE) interactions, the Valencia model for a multinucleon meson exchange current contribution [21] and the Rein-Seghal model [22] for single-pion production. The deep-inelastic-scattering channel is simulated using the GRV98 [23] parton distribution functions with BodekYang corrections [24] for the cross section, while hadron production is modeled by either PYTHIA5.72 [25] or a custom model based on KNO scaling (see the presentation in Sec. V.C of Ref. [26]) for interactions with a hadronic invariant mass above and below $2 \mathrm{GeV}$ respectively. It should be noted that the CCQE model contains both a mean-field and a "short range correlation" (SRC) component, the latter of which produces two outgoing nucleons. Final state interactions (FSI) of hadrons are described using cascade models tuned to hadron-nucleus scattering data $[16,27]$. This is the same neutrino interaction model that is used in the last T2K oscillation analysis and described in details in Ref. [12]. NUISANCE [28] is used to process the NEUT output. In total 6 million neutrino interactions and 2 million antineutrino interactions are simulated, corresponding to an estimated $3.0 \times 10^{22}$ and $4.5 \times 10^{22}$ POT, respectively. The simulation is scaled to test sensitivity as a function of accumulated statistics assuming 1.9 tons of mass in the Super-FGD fiducial volume.

The parametrization of detector effects is applied for protons, neutrons, muons and charged pions on a particleby-particle basis. For charged particles a Gaussian momentum and angular smearing is applied alongside a probability to not reconstruct the particle (to model inefficiencies). These response functions are applied based on a particle's type and as a function of true momentum and direction. Neutron resolutions and efficiencies are also applied and

\footnotetext{
${ }^{1}$ Further reconstruction efforts will aim to recover some of this lost selection efficiency.
}
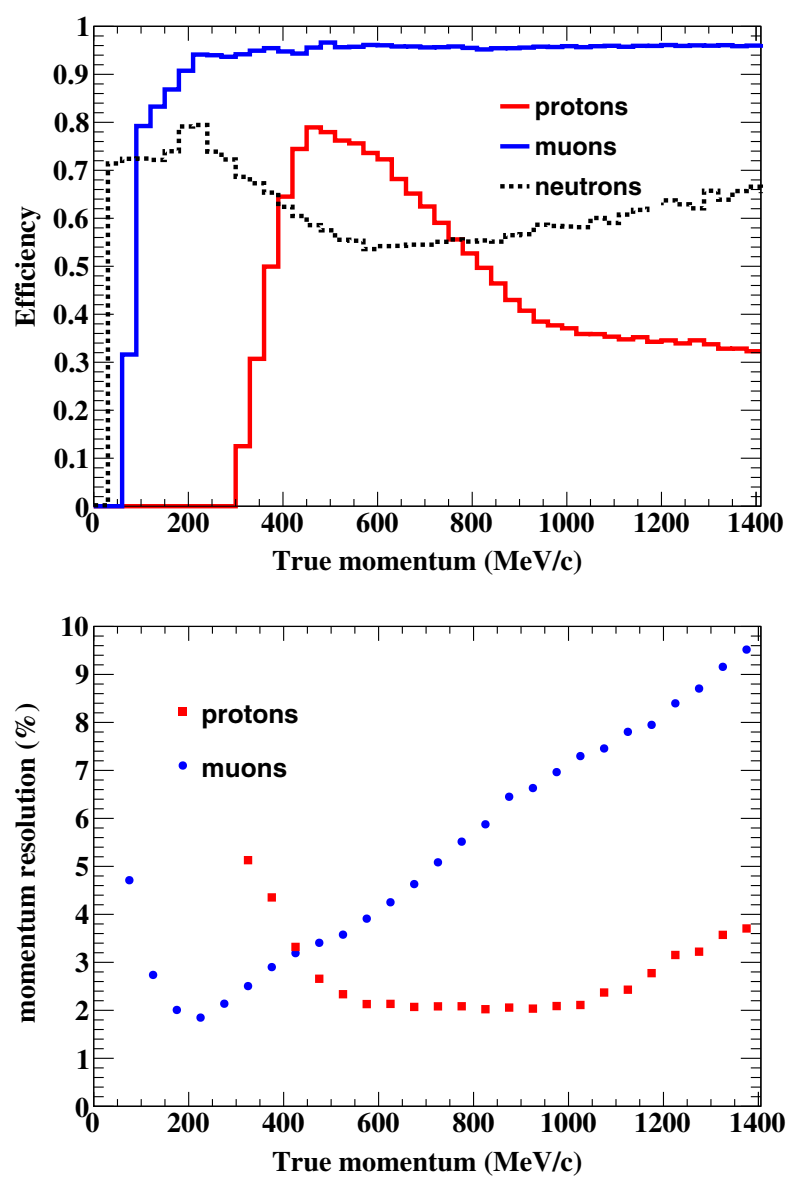

FIG. 2. Top: the efficiency to detect protons, muons and neutrons and bottom: the momentum resolution of muons and protons as a function of their respective momenta. The neutron momentum resolution ranges from $\sim 15 \%$ to $30 \%$ and is discussed in more detail in Ref. [13].

are handled as described in Ref. [13] (using the method where the time resolution depends on the light yield within a cube). In this analysis no cut is made on the distance the neutron travels from the interaction vertex (i.e., no "leverarm" cut on the neutron propagation distance is applied), which increases neutrino detection efficiency but also degrades the momentum resolution. The modeled detector performance is summarized in Fig. 2, which describes the momentum resolution and selection efficiency for muons, protons and neutrons. The decrease in proton selection efficiency after $500 \mathrm{MeV} / \mathrm{c}$ is largely from track rejection due to identified secondary interactions, but at higher momentum $(>1 \mathrm{GeV} / \mathrm{c})$ track rejection from ambiguous PID also plays a role. The parabolic shape of the resolutions stems from difficulties in reconstructing very short tracks, followed by peak performance for fully contained tracks, while higher momentum tracks reach the TPCs with relative resolution worsening at higher momentum (as expected due to the smaller curvature in the magnetic field).

Table I shows the number of reconstructed events in a sample of charged-current interactions without 
TABLE I. Number of reconstructed events in $\mathrm{CC} 0 \pi$ channels for $1 \times 10^{22}$ protons on target in neutrino mode and $1 \times 10^{22}$ in antineutrino mode.

\begin{tabular}{lcc}
\hline \hline Channel & Neutrino events & Antineutrino events \\
\hline CCQE & 299822 & 109750 \\
2p2h & 63010 & 19116 \\
Pion absorption & 48966 & 6714 \\
Undetected pions & 36200 & 5828 \\
\hline \hline
\end{tabular}

reconstructed pions in the final state $(\mathrm{CC} 0 \pi)$ and with at least one reconstructed proton/neutron for neutrino/antineutrino interactions. In this study we consider only these $\mathrm{CC} 0 \pi$ selections, which is the dominant interaction topology for $\mathrm{T} 2 \mathrm{~K}$ oscillation analyses. For reference, the current ND280 would expect to select $\sim 76,000$ neutrino interactions with at least one reconstructed proton in the final state for $1 \times 10^{22}$ POT [29] and has not been shown to be able to reconstruct neutrons.

It should be noted that this simple parametrized approach cannot account for all the physics of a full reconstruction and event selection. Most importantly neutral pions are assumed to be always rejected and the possibility of misidentifying one particle type as another is not considered beyond the aforementioned impact on the selection efficiency. While these are important limitations, in general they are subdominant effects for the $\mathrm{CC} 0 \pi$ selections considered in this paper. Current ND280 CC0 $\pi$ selections with a reconstructed proton in the final state have around $20 \%$ background fully dominated by undetected pions, only a small minority of this background stems from a misidentification of particle type or from an undetected neutral pion $[12,29]$.

\section{B. Analysis strategy}

The T2K oscillation analysis (see e.g., Ref. [12]) relies on a fit of ND280 data to constrain a parametrized model of the neutrino flux and interaction cross sections. The primary ND280 samples are split depending on whether zero, one or more charged pions are reconstructed in the final state. The distribution of muon kinematics (momentum and angle) in each sample is then compared to the model, thereby providing constraints on the parameters. The results of applying the same analysis to a sample of events simulated in ND280 Upgrade are shown in Ref. [4]. In this paper a similar strategy is applied for the $\mathrm{CC} 0 \pi$ channel and one nucleon in the final state only, but now fitting data presented as a function of hadronic kinematics and its correlations with lepton kinematics, which better exploits the performance assets of the Super-FGD.

Various observables for characterizing the hadronic part of the final state have been investigated in neutrino crosssection measurements at ND280 [29], and other experiments (e.g., MINERvA [30]). These include measurements of proton multiplicity and kinematics as well as of correlations between outgoing protons and muons [31,32], which

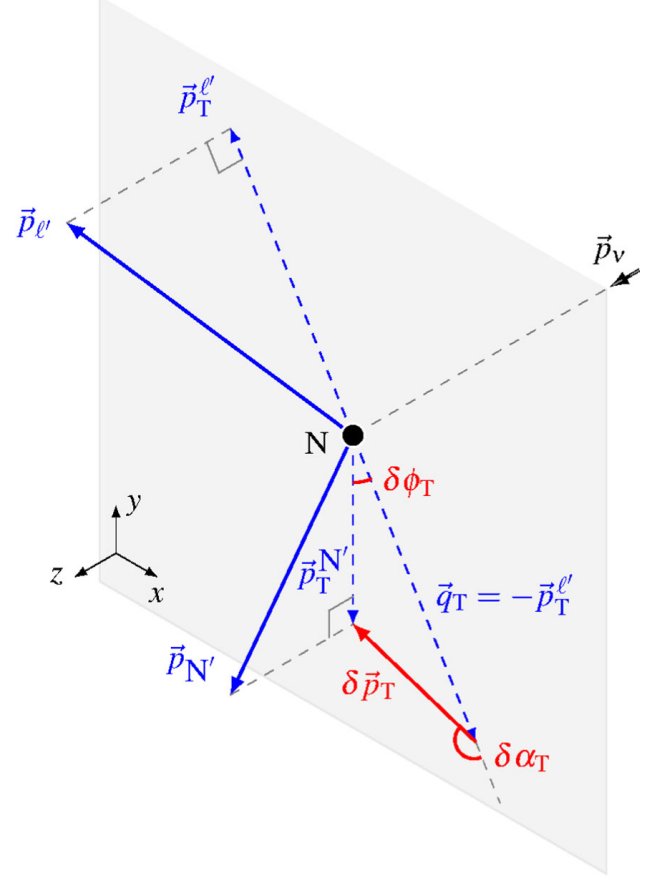

FIG. 3. A schematic illustration showing the definition of the variables describing transverse kinematic imbalance employed within this work. The incoming neutrino momentum vector is shown in black (defined to be along the $\mathrm{z}$-axis) while the outgoing lepton $\left(\ell^{\prime}\right)$ and highest momentum nucleon $\left(N^{\prime}\right)$ momentum vectors are shown as blue solid lines while their projection onto the $\mathrm{x}-\mathrm{y}$ plane, transverse to the incoming neutrino, are shown as dashed lines. The resultant triangle formed from these transverse projections defines the two variables considered within this work: $\delta \alpha_{\mathrm{T}}$ and $\delta p_{\mathrm{T}}$ (the magnitude of $\delta \vec{p}_{\mathrm{T}}$ shown in the figure). The figure is reused from Ref. [31].

are particularly sensitive to the nuclear effects that often drive the dominant uncertainties in neutrino oscillation analyses [1]. The improved performances of ND280 Upgrade in the measurements of such variables are shown in Ref. [4]. In this analysis a fit to some of these variables is performed to estimate ND280 Upgrade's potential to constrain flux and neutrino-interaction systematic uncertainties for future oscillation analyses. As detailed in the following subsection, the "transverse boosting angle" $\left(\delta \alpha_{T}\right)$ and the magnitude of the transverse momentum imbalance between the outgoing lepton and nucleon $\left(\delta p_{\mathrm{T}}\right)$ are chosen for this work, which are defined schematically in Fig. 3. These variables analyze the size and direction of the momentum imbalance between the outgoing lepton and nucleon on the plane transverse to the incoming neutrino beam [31].

It should be highlighted that the optimal analysis with ND280 Upgrade would be a multidimensional fit including the muon kinematics, the hadronic kinematics and their correlations. The tools to implement such an approach are under development within T2K. In this paper the study is limited to fitting pairs of variables at once, focusing only on 


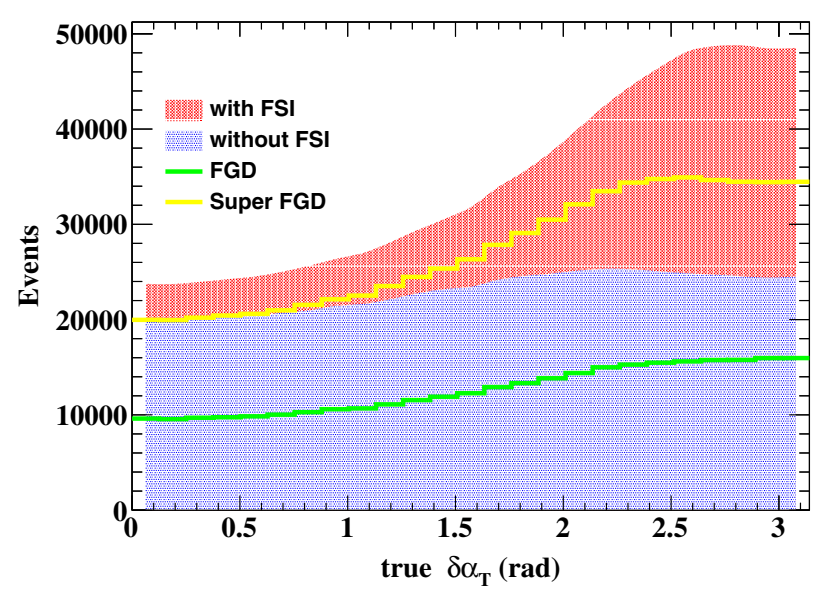

FIG. 4. The shaded regions show the generator level $\delta \alpha_{T}$ distribution for all $\mathrm{CC} 0 \pi$ interactions for $1 \times 10^{22}$ POT split by whether or not the outgoing nucleon underwent FSI. The overlaid solid lines indicate how the total $\delta \alpha_{T}$ distribution (including the with and without FSI components) changes when imposing the current ND280 FGD proton tracking threshold $(450 \mathrm{MeV} / \mathrm{c})$ and expectation from the Super-FGD (300 MeV/c).

those which exploit the lepton-nucleon correlations. The results are therefore highlighting only the additional sensitivity that such new variables could provide, with respect to the existing $\mathrm{T} 2 \mathrm{~K}$ analyses.

\section{Fit variables}

The inclusion of nucleon kinematics into the analysis requires a strategy to keep the nucleon FSI under control. In future oscillation analyses this will act as a new nuisance uncertainty which must be understood in order to propagate constraints on other relevant nuclear effects from the near to far detectors. The shape of $\delta \alpha_{T}$ has been shown to be particularly sensitive to nucleon FSI [31] and is therefore chosen as one of the fit variables for this study. In Fig. 4, the $\delta \alpha_{T}$ distribution for neutrino interactions is shown for $\mathrm{CC} 0 \pi$ events with and without FSI. As shown, in the absence of FSI the $\delta \alpha_{T}$ distribution is expected to be almost flat while with FSI an enhancement at large $\delta \alpha_{T}$ is predicted (stemming from the fact that FSI tends to slow down the outgoing nucleons). Previous analyses of $\delta \alpha_{T}$ at ND280 have not been able to clearly identify this FSI induced enhancement because of its high proton tracking threshold $(\sim 450 \mathrm{MeV} / \mathrm{c})$ which excludes many interactions in which the nucleon underwent FSI $[29,33]$. This is illustrated in Fig. 4, which shows how the current ND280 tracking threshold flattens $\delta \alpha_{T}$ to a greater extent than that of ND280 Upgrade. As discussed in Sec. I, it can also be noted that the neutron in antineutrino interactions can be reconstructed, through its time of flight, down to very low momentum threshold. This therefore potentially allows a useful FSI constraint even in the very low neutron momentum regions that cannot be measured for protons in the corresponding neutrino interactions [13].

Some of the most important systematic uncertainties for CCQE interactions in neutrino oscillation analyses are those which cause a bias in estimators for reconstructing neutrino energy. Such a bias directly impacts the measurement of neutrino oscillation parameters, notably $\Delta m_{32}^{2}$ and $\delta_{C P}$ precision measurements, especially in the presence of large $C P$ violation. The principle cause of this bias stems from: the modeling of the initial target nucleon momentum within the nucleus (Fermi motion); the contribution of CCnon-QE or SRC processes entering $\mathrm{CC} 0 \pi$ samples; and the "removal" energy it takes to liberate target nucleons from the nucleus (the latter was responsible for a dominant systematic uncertainty in T2K's latest neutrino oscillation analysis [12]). $\delta p_{T}$ [31] and $p_{N}^{2}$ (an extension to $\delta p_{\mathrm{T}}$ considering also an estimation of the longitudinal momentum imbalance in order to estimate the initial state nucleon momentum) [32] are known to well separate CCQE from $\mathrm{CC}-$ non-QE in $\mathrm{CC} 0 \pi$ samples while also well characterizing the Fermi motion. In antineutrino interactions these variables can also separate the hydrogen and carbon contributions of the $\mathrm{CH}$ scintillator, thereby allowing some lifting of the degeneracy between nucleon and nuclear level effects, while also providing the opportunity for improved in-situ flux constraints [13]. The reconstructed distribution of $\delta p_{T}$ for neutrino and antineutrino interactions are shown in Fig. 5. The neutrino case demonstrates the clear separation of CCQE in the bulk and CC-non-QE in the tail (the small CCQE contribution to the tail is from SRCs and nucleon FSI). The antineutrino distribution does not show such good mode separation (due to the relatively poor neutron momentum resolution) but the shape difference of the hydrogen and carbon contributions is clearly visible.

While variables characterizing transverse kinematic imbalance are sensitive to many of the most important systematic uncertainties for neutrino oscillation analyses, they are not particularly sensitive to nuclear removal energy effects. A constraint can instead be established by looking for systematic shifts from expectation in the reconstructed neutrino energy distribution. However, in order to strongly constrain the removal energy uncertainty, a very good resolution is necessary in the reconstruction of neutrino energy at the near detector, together with a very good control of energy scale in the detector and of the flux energy peak.

The usual estimator of neutrino energy is based on the very well-known (e.g., see Ref. [12]) formula for CCQE events, relying on muon kinematics only:

$$
E_{\mathrm{QE}}=\frac{m_{p}^{2}-m_{\mu}^{2}-\left(m_{n}-E_{B}\right)^{2}+2 E_{\mu}\left(m_{n}-E_{B}\right)}{2\left(m_{n}-E_{B}-E_{\mu}+p_{\mu}^{z}\right)},
$$

\footnotetext{
${ }^{2}$ Note that a subscript "N" (denoting nucleon) is used rather than " $n$ " (for neutron) since in these studies the variable can be calculated for neutrino or antineutrino interactions corresponding to probes of the initial state neutron or proton respectively.
} 

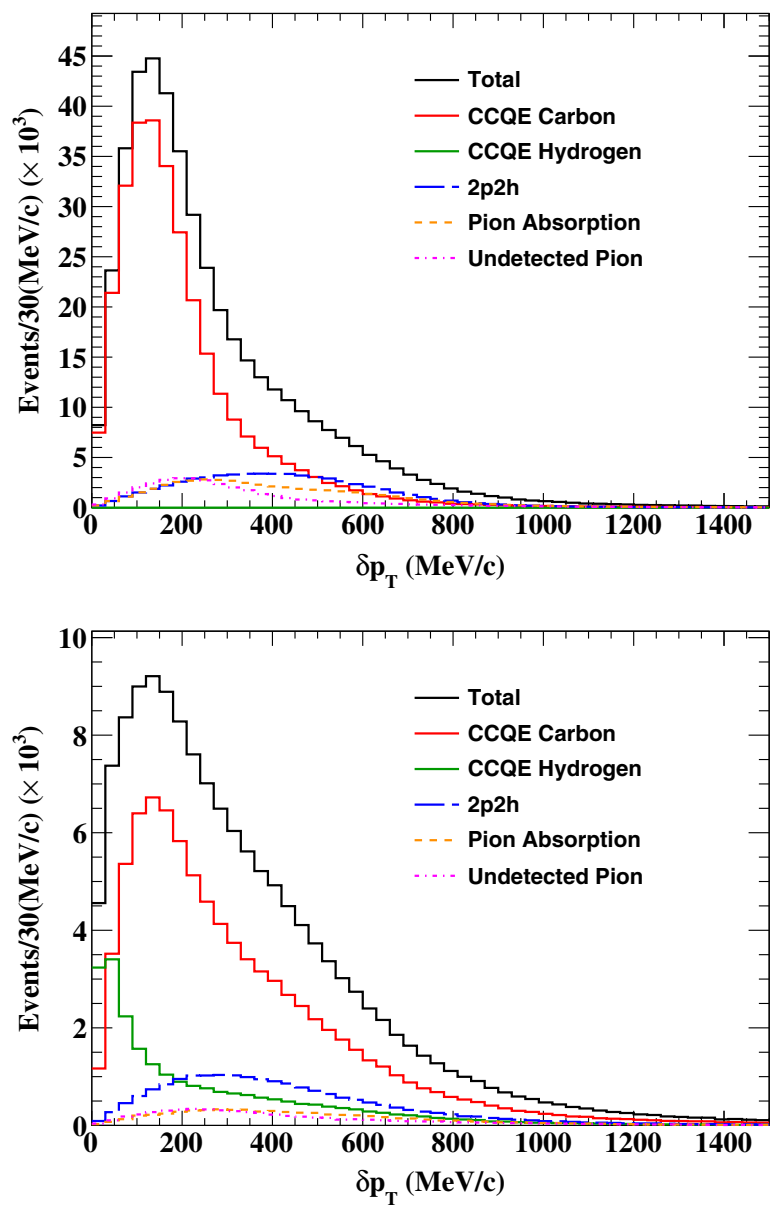

FIG. 5. The reconstructed $\delta p_{T}$ distribution for selected $\mathrm{CC} 0 \pi$ neutrino (upper) and antineutrino (lower) interactions split by interaction mode and target for $1 \times 10^{22}$ POT.

where $m_{p / \mu / n}$ is the mass of a proton/muon/neutron; $E_{\mu}$ and $p_{\mu}^{z}$ is the outgoing muon energy and momentum projected along the direction of the incoming neutrino; and $E_{B}$ is some assumed fixed nuclear binding energy of the struck nucleon (which is related to, but not exactly, the removal energy and is usually taken to be $\sim 25 \mathrm{MeV}$ for carbon $[34,35])$. A second estimator can be defined as:

$$
E_{\mathrm{vis}}=E_{\mu}+T_{N}
$$

where $T_{N}$ is the kinetic energy of the outgoing proton (neutron) in neutrino (antineutrino) interactions, $E_{\mu}$ is the total energy of the outgoing muon. $E_{\mathrm{vis}}$ is the total visible energy of all outgoing particles in $\mathrm{CC} 0 \pi$ events with one nucleon in the final state. Such an estimator, before detector smearing, is expected to be slightly smaller than the true neutrino energy in CCQE events due to the need to overcome the nuclear removal energy and the loss of energy through nucleon FSI. Similarly multinucleon interactions and pion absorption will populate the tail of low
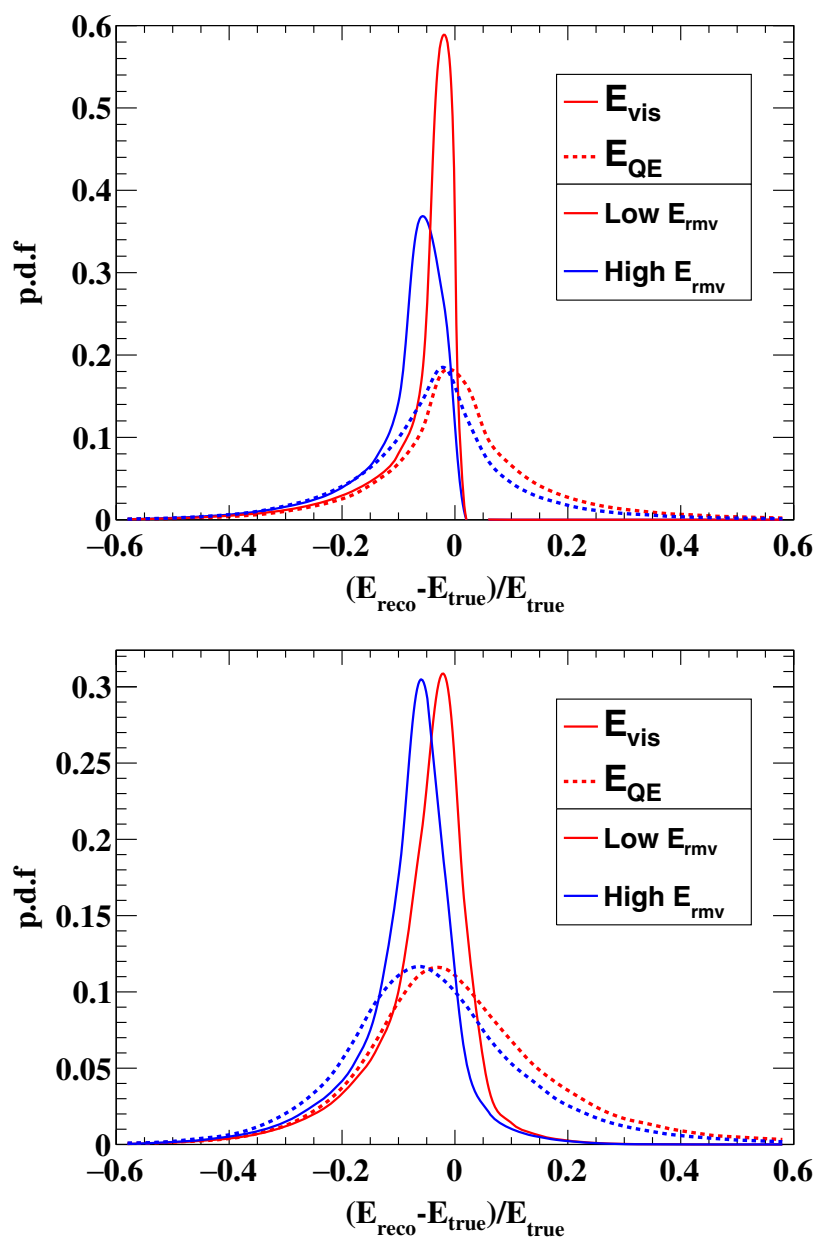

FIG. 6. The neutrino energy reconstruction resolution and bias is shown for the two estimators defined in the text $\left(E_{\mathrm{vis}}\right.$ and $E_{\mathrm{QE}}$ as solid and dashed lines respectively) for $\pm 10 \mathrm{MeV}$ shifts to the nominal removal energy $\left(E_{\mathrm{rmv}}\right)$ distribution (denoted by the red and blue colors respectively). The upper plot does not include the effect of detector smearing such that $E_{\text {reco }}$ is constructed using the true muon and proton kinematics directly from the generator. In the lower plot $E_{\text {reco }}$ is instead built from the corresponding reconstructed quantities (i.e., with the detector smearing applied).

$E_{\mathrm{vis}}$ since the second nucleon or the absorbed pion carry away some of the initial neutrino energy.

Figure 6 compares the neutrino energy resolution for the two estimators alongside the impact of a possible bias due to removal energy. The distributions at generator level and after detector effects are shown. The $E_{\text {vis }}$ estimator has a higher peak at very good resolution $(<5 \%)$ thus showing an increased sensitivity to possible bias in the removal energy estimation. This feature is preserved at reconstructed level, despite a larger experimental smearing of $E_{\text {vis }}$, due to the inclusion of proton tracking resolution in addition to the muon one. The observed smearing of $E_{\mathrm{vis}}$ in data will be therefore mostly due to detector effects, while the smearing of $E_{\mathrm{QE}}$ is dominated by Fermi motion. The smearing induced by tracking resolution can in principle be 
improved using better detectors. Moreover, we expect to be able to model quite precisely the detector-induced smearing, thanks to test beam studies and detector simulation, while the smearing due to nuclear effects is typically less well known. The inclusion of both energy estimators and the study of their correlation may also enable future enhanced sensitivity to removal energy.

To achieve a good sensitivity while keeping crosssection systematic uncertainties manageable, the pairs of variables used in the fit are one of the "single transverse variables," $\delta p_{T}$ or $\delta \alpha_{T}$, alongside the total visible energy (sum of the muon and nucleon energy in the final state). The aforementioned $p_{N}$ [32] variable is also considered as an alternative to $\delta p_{T}$ and is discussed in the Appendix A. The full set of input histograms are shown in Appendix B.

\section{Fit parameters}

The uncertainty model used in this analysis is designed to offer theory-driven conservative freedoms to modify pertinent aspects of the neutrino interaction model in addition to accounting for flux modeling and detector performance uncertainties. Particular care is taken to allow plausible variations of nucleon kinematics which are especially sensitive to nuclear effects. While this sensitivity is at the core of the improvements driven by ND280 Upgrade, it also requires a parametrization of nuclear uncertainties beyond what is used by the latest $\mathrm{T} 2 \mathrm{~K}$ oscillation analyses, which exploit only measurements of lepton kinematics.

For NEUT's CCQE interactions the initial nuclear state is characterized by a two-dimensional spectral function (SF) which describes the momentum of initial state nucleons and the energy that is necessary to remove them from the nucleus. In the SF model there is an extended distribution of removal energies rather than a fixed nuclear binding energy. The SF is broadly split into two parts: a part described by mean-field physics which has a shell structure (for carbon: one sharp p-shell and a diffuse s-shell) and a SRC part which results in two nucleons in the final state. The uncertainty model used allows a variation of the normalization of each of the two mean-field shells separately and of the total strength of the SRC component. A wide prior uncertainty of $30 \%$ is applied to the SRC and shell parameters. Further freedom is added through an overall removal energy shift parameter (similar to what is described in Ref. [12]) which is built using an interpolation between two alternative simulation histograms that are generated with opposite $10 \mathrm{MeV}$ shifts of the whole removal energy distribution. The histograms are constructed in the same binning used for the fit and in the same variables. The removal parameter is not constrained with any prior uncertainty. For antineutrino interactions off a hydrogen target nuclear effects are not relevant, but a 5\% normalization uncertainty is considered to account for imperfectly modeled nucleon-level effects.
The $2 \mathrm{p} 2 \mathrm{~h}$ uncertainties are provided through two normalization factors, one for $E_{\mathrm{vis}}<600 \mathrm{MeV}$ and one for the remaining phase space. This accounts for uncertainties in $2 \mathrm{p} 2 \mathrm{~h}$ nucleon ejection model, allowing $2 \mathrm{p} 2 \mathrm{~h}$ shape to change as a function of neutrino energy and a consistent treatment between fits with different pairs of variables. The $2 \mathrm{p} 2 \mathrm{~h}$ uncertainties are not constrained prior to the fit.

Since the pion production contribution to the selected samples is low, uncertainties on this are modeled via two parameters: one controlling the normalization of the pion production background (i.e., events in which a pion was not detected) and another to alter the normalization of the pion absorption FSI contribution. For a conservative approach, neither are constrained with prior uncertainties.

Nucleon FSI is treated similarly to the removal energy shift parameter, relying on an interpolation between histograms generated with NEUT using different nucleon FSI strengths. These are constructed by rerunning NEUT with 30\% modifications of nucleon mean free paths inside the FSI cascade to cover differences between different FSI models and the variation in nuclear transparency data $[36,37]$.

The flux uncertainties are handled using the T2K flux covariance matrix shown in Ref. [18], which provides correlated uncertainties on the flux between ranges of neutrino energy. Flux shape uncertainties are a second order effect, and so for computational ease the covariance is applied to bins of visible energy rather than true neutrino energy. In this way only flux uncertainties covering energies between 0.2 and $1.5 \mathrm{GeV}$ are included. The range starts from $0.2 \mathrm{GeV}$ since this is the starting point of $E_{\mathrm{vis}}$ histogram, but this covers the ND280 flux peak and the primary region of interest for neutrino oscillation analysis.

We additionally consider a "bin-to-bin uncorrelated uncertainty" which accounts for systematic uncertainties in detector response modeling and small shape freedoms not included via the other parameters. Rather than existing as a fit parameter, this uncertainty is included by directly modifying the likelihood to effectively add an uncorrelated additional uncertainty to every bin of the analysis. Based on the size of detector uncertainties and background model shape parameters in T2K measurements of $\delta p_{T}$ [29], this is chosen to be $11.6 \%$ at $6 \times 10^{21}$ POT and is then scaled up or down with the square root of the POT to represent improved constraints on the corresponding systematic uncertainties.

The prior uncertainties within the systematic penalty term, discussed in Sec. II B 3, and the bin-to-bin uncorrelated uncertainty are summarized in Table II.

\section{Fitter details}

A binned likelihood fitter is built in two-dimensions, including statistical and systematic uncertainties. The statistical uncertainty is implemented as a Poisson term in the likelihood while the systematic uncertainties are 
TABLE II. A list of fit parameters, their prior constraints and notes regarding their application. While not a fit parameter, the bin-to-bin uncorrelated uncertainty is also listed.

\begin{tabular}{lcc}
\hline \hline Parameter & Prior Constraint & Notes \\
\hline $\begin{array}{l}\text { p-shell } \\
\text { normalization }\end{array}$ & $30 \%$ \\
s-shell & \\
$\quad$ normalization & $30 \%$ \\
SRC strength & $30 \%$ & \\
total QE & $10 \%$ & \\
$\quad$ normalization & & \\
Removal energy & Unconstrained & \\
$\quad$ shift & & \\
2p2h, low & Unconstrained & \\
2p2h, high & Unconstrained & \\
Undetected pions & Unconstrained & \\
Pion FSI & Unconstrained & \\
$\quad$ contribution & & \\
Nucleon FSI & $30 \%$ & \\
$\quad$ strength & & \\
Flux (binned $E_{\text {vis }}$ ) & T2K covariance & \\
Hydrogen & $5 \%$ & \\
$\quad$ normalization & & \\
Uncorrelated & $11.6 \%$ (at & No parameter fit, POT \\
Uncertainty & $6 \times 10^{21}$ POT) & dependence \\
\hline \hline
\end{tabular}

parametrized as a function of the fit variables and implemented as nuisances with priors included mostly as Gaussian penalty terms in the likelihood. The prior uncertainties and fit variables are those discussed in Sec. II B 2. One exception to the treatment of the prior uncertainties is an ad-hoc bin-to-bin uncorrelated uncertainty (as also detailed in Sec. II B 2) which is added directly to the likelihood using the Barlow-Beeston approach [38]. The final $\chi^{2}$ used in the fitter is therefore defined as:

$$
\begin{aligned}
\chi^{2}= & \sum_{i=1}^{n \text { bins }} 2\left(\beta_{i} E_{i}-O_{i}+O_{i} \ln \frac{O_{i}}{\beta_{i} E_{i}}+\frac{\left(\beta_{i}-1\right)^{2}}{2 \delta^{2}}\right) \\
& +\sum_{j}\left(\frac{p_{j}^{\prime}-p_{j}}{\sigma_{j}}\right)^{2}, \\
\beta_{j}= & \frac{1}{2}\left(-\left(E_{i} \delta^{2}-1\right)+\sqrt{\left(E_{i} \delta^{2}-1\right)^{2}+4 O_{i} \delta^{2}}\right),
\end{aligned}
$$

where the first term is from the Poisson likelihood (with the Barlow-Beeston extension) and the second term is the Gaussian penalty. The definition of the Barlow-Beeston scaling parameter $\beta$ is also given. $O_{i}$ and $E_{i}$ are the observed and expected number of events for bin $i, \delta$ is the size of the uncorrelated uncertainty included directly in the Poisson likelihood. The second term is a sum over the systematic parameters in the fit where $p_{j}^{\prime}$ and $p_{j}$ are the value of the parameter and its prior value respectively. $\sigma_{j}$ is the prior uncertainty of parameter $j$.
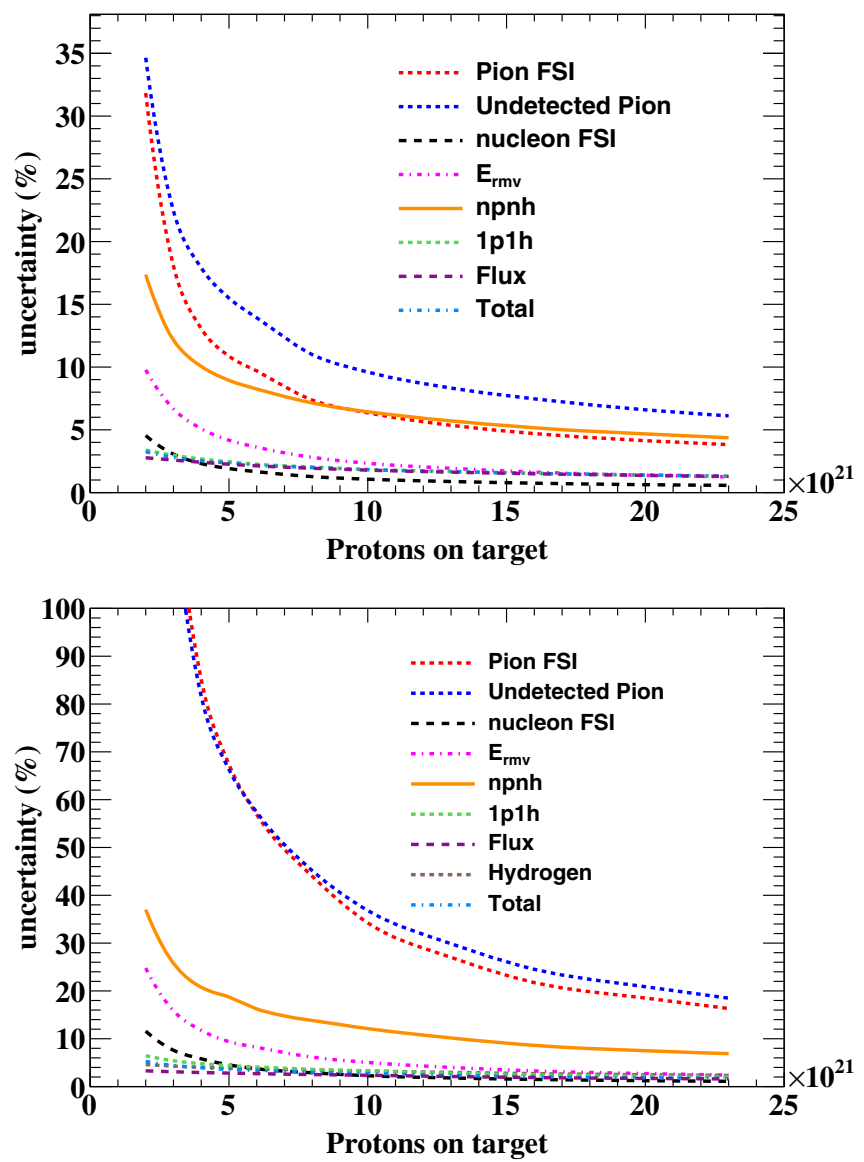

FIG. 7. The $1 \sigma$ sensitivity to systematic parameters as function of POT in neutrino case (top) and antineutrino case (bottom) when fitting the reconstructed $\mathrm{CC} 0 \pi$ data binned in $\delta p_{T}$ and $E_{\mathrm{vis}}$. The values in the plot are the ratio of the parameter uncertainty to the parameter nominal value expressed as a percentage.

\section{RESULTS AND DISCUSSION}

The fit described in Sec. II is performed and the parameter uncertainties obtained are evaluated as a function of the statistics accumulated (denoted by the simulated POT exposure). Note that all the systematic uncertainties are always fit together, even if the uncertainty on only one parameter is shown. A summary of the results are shown in Figs. 7 and 8, which shows parameter constraints as a function of POT and the correlations between fit parameters (for $10^{22}$ POT) respectively. In the former figure the parameters describing CCQE and $2 \mathrm{p} 2 \mathrm{~h}$ interactions are integrated to provide more meaningful uncertainties on the total one-particle-one-hole $(1 \mathrm{p} 1 \mathrm{~h})$ final state normalization (CCQE without SRCs) and an accompanying npnh final state normalization (2p2h and CCQE with SRCs). SRCs are combined with $2 \mathrm{p} 2 \mathrm{~h}$ since they have similar kinematic properties (as can be seen by their anticorrelation) and both are responsible for similar neutrino energy reconstruction bias. The uncertainty on the total cross section (integrating all cross-section systematic parameters but not the flux) is 

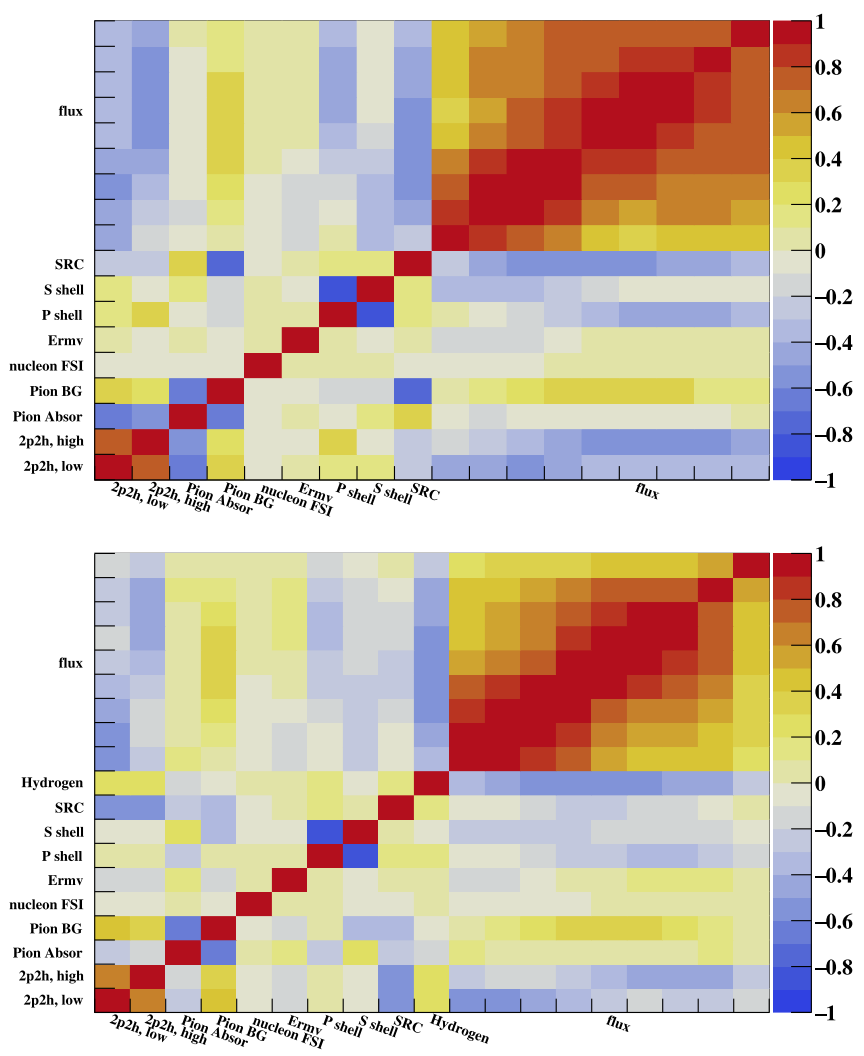

FIG. 8. The correlation matrix between constrained parameters following a fit to reconstructed $\mathrm{CC} 0 \pi$ data binned in $\delta p_{T}$ and $E_{\mathrm{vis}}$ with $1 \times 10^{22}$ POT. The results following a fit to neutrino (top) and antineutrino (bottom) samples are shown.

also shown. Tabulated sensitivities are shown for two fixed POT values in Table III.

As discussed in Sec. II B 1, it is expected that the SuperFGD should allow a particularly strong constraint on nucleon FSI via a measurement of $\delta \alpha_{T}$. To evaluate this, Fig. 9 shows only the nucleon FSI strength uncertainty as a function of the number of POT following fits using either $\delta \alpha_{T}$ or $\delta p_{T}$ alongside $E_{\mathrm{vis}}$. The extracted uncertainty on the nucleon FSI parameter is of the order of few percent at low statistics and can reach $1 \%$ with Hyper-K-era statistics. As can be seen, $\delta p_{T}$ is only slightly less sensitive to FSI than $\delta \alpha_{T}$, but such sensitivity comes from the tail of the distribution with some degeneracy with the impact of non-QE components and SRCs. As such, the FSI sensitivity in $\delta p_{T}$ is more dependent on the shape uncertainties assumed for non-QE and is less robust than the sensitivity from $\delta \alpha_{T}$. On the other hand we expect a full multidimensional fit, using both $\delta p_{T}$ and $\delta \alpha_{T}$, to provide an even more robust constraint on FSI and to be able to cross-check the correctness of FSI simulations through the investigation of possible tensions between the two variables.

For an antineutrino fit it is found that the benefit of using $\delta \alpha_{T}$ rather than $\delta p_{T}$ to constrain nucleon FSI is only realized after $\sim 6 \times 10^{21} \mathrm{POT}$, and the difference in sensitivity is reduced to the level of only $\sim 0.2 \%$. This is likely
TABLE III. Expected $1 \sigma$ uncertainties on key cross-section parameters at $1 \times 10^{22}$ POT (left) and $2 \times 10^{22}$ POT (right) for neutrino and antineutrino interactions with different fit variables.

\begin{tabular}{|c|c|c|c|}
\hline $1 \times 10^{22} \mathrm{POT}$ & $\delta p_{T} ; E_{\mathrm{vis}}$ & $\delta \alpha_{T} ; E_{\mathrm{vis}}$ & $p_{N} ; E_{\mathrm{vis}}$ \\
\hline 1p1h $(\nu)$ & $1.9 \%$ & $1.8 \%$ & $1.5 \%$ \\
\hline 1p1h $(\bar{\nu})$ & $3.3 \%$ & $3.9 \%$ & $2.6 \%$ \\
\hline npnh $(\nu)$ & $6.5 \%$ & $13 \%$ & $5.3 \%$ \\
\hline npnh $(\bar{\nu})$ & $12 \%$ & $17 \%$ & $11 \%$ \\
\hline$E_{r m v}(\nu)$ & $0.55 \mathrm{MeV}$ & $0.38 \mathrm{MeV}$ & $0.53 \mathrm{MeV}$ \\
\hline$E_{r m v}(\bar{\nu})$ & $1.3 \mathrm{MeV}$ & $1.0 \mathrm{MeV}$ & $1.3 \mathrm{MeV}$ \\
\hline Pion FSI $(\nu)$ & $6.6 \%$ & $14 \%$ & $4.8 \%$ \\
\hline Pion FSI $(\bar{\nu})$ & $34 \%$ & $35 \%$ & $30 \%$ \\
\hline Undetected pions $(\nu)$ & $9.7 \%$ & $14 \%$ & $8.2 \%$ \\
\hline Undetected pions $(\bar{\nu})$ & $37 \%$ & $36 \%$ & $31 \%$ \\
\hline Nucleon FSI $(\nu)$ & $1.1 \%$ & $0.76 \%$ & $0.98 \%$ \\
\hline Nucleon FSI $(\bar{\nu})$ & $2.3 \%$ & $1.9 \%$ & $2.4 \%$ \\
\hline Flux $(\nu)$ & $1.8 \%$ & $1.9 \%$ & $1.6 \%$ \\
\hline Flux $(\bar{\nu})$ & $2.4 \%$ & $2.3 \%$ & $2.2 \%$ \\
\hline Total $(\nu)$ & $1.8 \%$ & $2.1 \%$ & $1.6 \%$ \\
\hline Total $(\bar{\nu})$ & $2.7 \%$ & $2.7 \%$ & $2.5 \%$ \\
\hline Hydrogen $(\bar{\nu})$ & $3.3 \%$ & $4.0 \%$ & $2.9 \%$ \\
\hline $2 \times 10^{22} \mathrm{POT}$ & $\delta p_{T} ; E_{\mathrm{vis}}$ & $\delta \alpha_{T} ; E_{\mathrm{vis}}$ & $p_{N} ; E_{\mathrm{vis}}$ \\
\hline 1p1h $(\nu)$ & $1.4 \%$ & $1.2 \%$ & $1.1 \%$ \\
\hline 1p1h $(\bar{\nu})$ & $2.2 \%$ & $2.5 \%$ & $1.7 \%$ \\
\hline npnh $(\nu)$ & $4.7 \%$ & $8.5 \%$ & $3.6 \%$ \\
\hline npnh $(\bar{\nu})$ & $7.5 \%$ & $10.0 \%$ & $7.1 \%$ \\
\hline$E_{r m v}(\nu)$ & $0.32 \mathrm{MeV}$ & $0.22 \mathrm{MeV}$ & $0.3 \mathrm{MeV}$ \\
\hline$E_{r m v}(\bar{\nu})$ & $0.68 \mathrm{MeV}$ & $0.57 \mathrm{MeV}$ & $0.68 \mathrm{MeV}$ \\
\hline Pion FSI $(\nu)$ & $4.2 \%$ & $8.3 \%$ & $2.8 \%$ \\
\hline Pion FSI $(\bar{\nu})$ & $19 \%$ & $17 \%$ & $16 \%$ \\
\hline Undetected pions $(\nu)$ & $6.6 \%$ & $7.9 \%$ & $5.4 \%$ \\
\hline Undetected pions $(\bar{\nu})$ & $21 \%$ & $19 \%$ & $18 \%$ \\
\hline Nucleon FSI $(\nu)$ & $0.62 \%$ & $0.43 \%$ & $0.58 \%$ \\
\hline Nucleon FSI $(\bar{\nu})$ & $1.2 \%$ & $0.98 \%$ & $1.2 \%$ \\
\hline Flux $(\nu)$ & $1.4 \%$ & $1.4 \%$ & $1.2 \%$ \\
\hline Flux $(\bar{\nu})$ & $1.7 \%$ & $1.6 \%$ & $1.6 \%$ \\
\hline Total $(\nu)$ & $1.4 \%$ & $1.5 \%$ & $1.2 \%$ \\
\hline Total $(\bar{\nu})$ & $1.8 \%$ & $1.7 \%$ & $1.7 \%$ \\
\hline Hydrogen $(\bar{\nu})$ & $2.5 \%$ & $3.1 \%$ & $2.1 \%$ \\
\hline
\end{tabular}

mainly due to the lower resolution and less statistics per POT than in the neutrino fit, but it should be noted that using $\delta p_{T}$ in the antineutrino fit also allows a more independent constraint on the neutrino flux, as discussed in Sec. II B 1, and this benefit may partially offset the degeneracies lifted by $\delta \alpha_{T}$.

Figure 10 shows the constraint on the removal energy parameter from a fit to $E_{\mathrm{vis}}$ and $\delta p_{T}$ for neutrino and antineutrino interactions. In the neutrino case the removal energy shift can be measured at $2 \mathrm{MeV}$ at relatively low statistics and better than $1 \mathrm{MeV}$ with ultimate statistics. The corresponding antineutrino constraint is 3 to 4 times worse. As discussed in Appendix A, further improved constraints can be obtained by exploiting the $p_{N}$ variable in place of $\delta p_{T}$, but in this case more longitudinal information is 

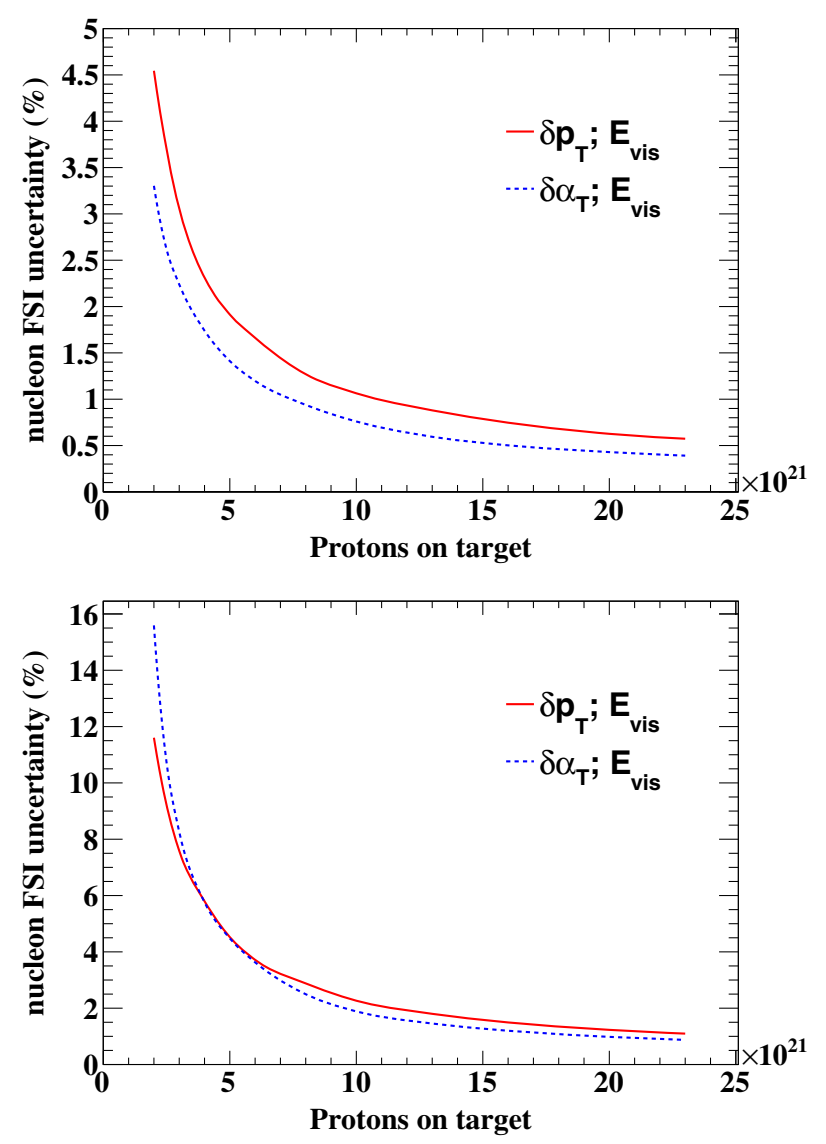

FIG. 9. The $1 \sigma$ sensitivity to the nucleon FSI parameter as a function of POT for neutrino interactions when fitting the reconstructed $\mathrm{CC} 0 \pi$ data binned in $\delta p_{T}$ and $E_{\mathrm{vis}}$ or in $\delta \alpha_{T}$ and $E_{\text {vis }}$. The results are shown for neutrino (top) and antineutrino (bottom) case.

included and thus the constraint is more reliant on the neutrino flux shape prediction.

As discussed in Sec. II B 1, non-QE and SRC interactions in the $\mathrm{CC} 0 \pi$ channel can induce an important bias

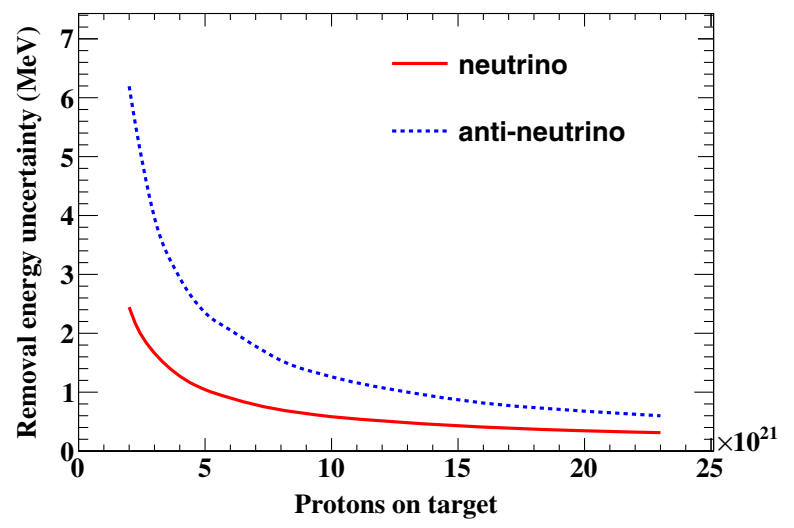

FIG. 10. The $1 \sigma$ sensitivity to the nuclear removal energy shift parameter as a function of POT for neutrino and antineutrino interactions when fitting the reconstructed $\mathrm{CC} 0 \pi$ data binned in $\delta p_{T}$ and $E_{\text {vis }}$.
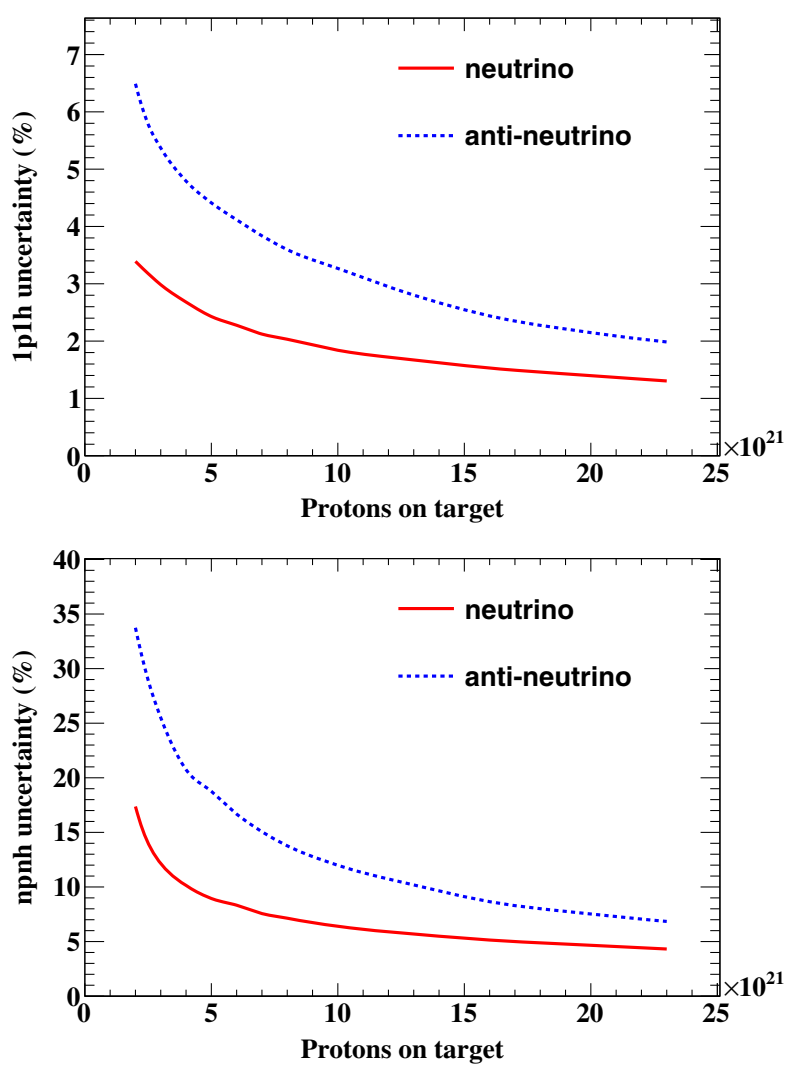

FIG. 11. The $1 \sigma$ sensitivity to the $1 \mathrm{p} 1 \mathrm{~h}$ (top) and npnh (bottom) cross-section normalizations as a function of POT for neutrino and antineutrino interactions when fitting the reconstructed $\mathrm{CC} 0 \pi$ data binned in $\delta p_{T}$ and $E_{\text {vis }}$.

in the reconstruction of neutrino energy. Figure 11 shows the constraint on the total normalization of $1 \mathrm{p} 1 \mathrm{~h}$ and npnh interactions. As previously noted, relatively advanced shape uncertainties are considered for these processes in the fit but for brevity we show only their combined effect on the total cross sections. The bulk of the $\delta p_{T}$ variable is a direct probe of the $1 \mathrm{p} 1 \mathrm{~h}$ interactions, allowing a constraint as good as $\sim 1.5 \%(\sim 2 \%)$ in neutrino (antineutrino) interactions. The tail at high values of $\delta p_{T}$ is sensitive to the non-QE component, enabling a constraint better than $5 \%(10 \%)$ in neutrino (antineutrino) interactions. The npnh constraint is partially correlated with the constraint on the pion absorption FSI component and the background due to undetected pions in the final state. It should be noted that the effects of such components on the neutrino energy reconstruction are similar so induce similar bias in the neutrino oscillation parameters. Indeed, pion FSI and the $2 \mathrm{p} 2 \mathrm{~h}$ process including a $\Delta$ resonance excitation and a pion production followed by reabsorption, are separately modeled in NEUT but they are fundamentally very similar processes (i.e., they are partially irreducible backgrounds to each other). On the contrary, the background due to the presence of an undetected pion in the final state can be reduced, notably thanks to the lower threshold for pion reconstruction in the Super-FGD. 

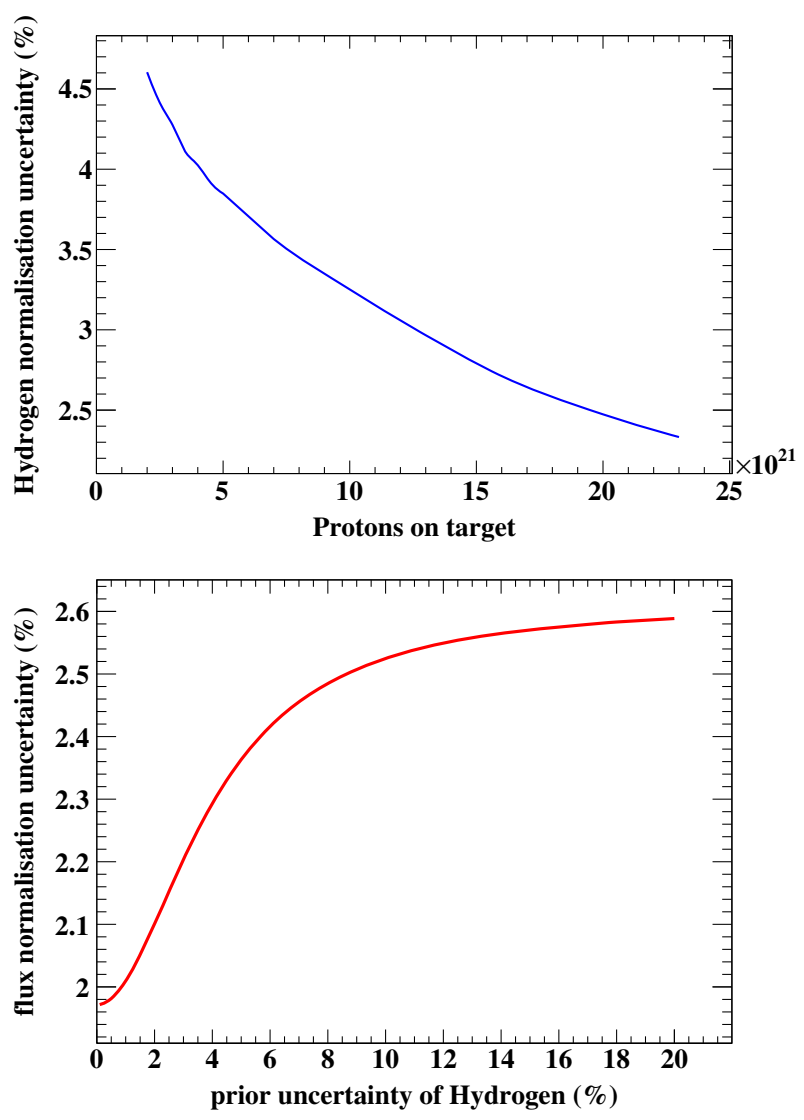

FIG. 12. The $1 \sigma$ sensitivity to the hydrogen normalization parameter as a function of POT (top) and the $1 \sigma$ sensitivity to the flux normalization as a function of the hydrogen parameter's prior uncertainty (bottom) for antineutrino interactions when fitting the reconstructed $\mathrm{CC} 0 \pi$ data binned in $\delta p_{T}$ and $E_{\mathrm{vis}}$ for $1 \times 10^{22}$ POT.

The main effect limiting the precision of the extrapolation of the $1 \mathrm{p} 1 \mathrm{~h}$ normalization from the near to the far detector is the degeneracy between the cross section and the neutrino flux. In Ref. [13], a strategy to select an hydrogen enhanced sample in antineutrino interactions has been proposed, in order to constrain the flux independently of uncertainties on nuclear effects. Here we consider the hydrogen sample together with the carbon sample in a joint fit to estimate quantitatively its impact on the flux constraints. The contribution of hydrogen interactions in the antineutrino sample is illustrated in Fig. 5 for the $\delta p_{T}$ distribution.

Figure 12 shows the constraint on the total normalization of the hydrogen sample, which is reduced by a factor of $\sim 2$ relative to the prior uncertainty with $2 \times 10^{22} \mathrm{POT}$ worth of data, in addition to the constraint on the flux normalization. The impact on the flux constraint depends on the prior uncertainties assumed on the antineutrino-nucleon cross section, notably due to the nucleon form factor. It should be noted that this is the result of a complete fit including both antineutrino-nucleus and antineutrino-nucleon processes, thus showing the incremental contribution to the flux precision from the hydrogen enhanced sample, on top of the constraint obtained from the usual fit to carbon interactions. The contribution of the hydrogen-enhanced sample is sizeable, if the prior hydrogen normalization uncertainty is lower than $\sim 10 \%$, improving the relative flux constraint by up to $\sim 20 \%$. Note that, while not quantified here, the power of the hydrogen-enhanced sample also improves knowledge of the flux shape and better separation between flux and form-factor constraints could be achieved by adding the reconstructed four-momentum $\left(Q^{2}\right)$ from the lepton kinematics as a fit variable. This would allow a separation of the low $Q^{2}$ region, in which the form factor is relatively well understood and so the flux can be constrained, from the high $Q^{2}$ region, where the prior flux constraint can instead be leveraged to probe the form-factor details.

\section{CONCLUSION}

It has been demonstrated that the upgraded ND280 detector will allow increasing sensitivity to nuclear-model uncertainties, enabled by the use of observables formed from both lepton and nucleon kinematics, which can complement the usual T2K analysis of only muon kinematics. Importantly, the use of nucleon kinematics also allows less room for incorrect models to describe ND280 Upgrade data and, as such, facilitates more robust constraints.

The inclusion of nucleon kinematics into the analysis brings new systematic uncertainties, notably detector systematics but also new nuclear-model systematics related with nucleon FSI. From simulated studies, benchmarked by test beam data of prototypes and by long-term data-taking experience with ND280, we expect the systematic uncertainties related with detector modelling to be well under control. In this paper we have shown quantitatively, for the first time, that nucleon FSI can also be very well constrained thanks to the use of $\delta \alpha_{T}$. The precision of the constraint is enabled by the low proton (and neutron) tracking threshold in ND280 Upgrade and the absence of degeneracy (correlation) with other nuclear-model uncertainties in $\delta \alpha_{T}$.

The use of an improved estimator of neutrino energy, based on the sum of muon energy and nucleon kinetic energy, has been investigated and shows interesting sensitivity to nuclear removal energy shifts. Furthermore, the $\mathrm{QE}$ and non-QE components of the cross section have been shown to be well separated by using fits to $\delta p_{T}$. Contrary to an inclusive analysis, these two components can be measured with small degeneracy/correlation, thus reducing ambiguities in the propagation of constraints to the far detector. Finally, the reduction of the flux uncertainty from a hydrogen-enhanced sample, as suggested in Ref. [13], has been quantified for the first time. The relative improvement on the flux normalization uncertainty can be up to $20 \%$, depending on the prior uncertainty on the nucleon form factors. 
Although a quantitative evaluation of the impact of ND280 Upgrade constraints directly on T2K and Hyper-K measurements of PMNS neutrino oscillation parameters is beyond the scope of this work, some qualitative observations can be made. The tight constraints on removal energy and npnh effects indicate a reduction of the uncertainty in both the neutrino energy scale and bias at the far detector. These effects are strongly correlated with the systematic uncertainty on measurements of the neutrino mass splitting $\left(\Delta m_{32}^{2}\right)$ and so a comparable reduction in the model systematic component of the uncertainty on its measurement might be expected. Measurements of the large mixing angle $\left(\theta_{23}\right)$ are more dependant on the total cross section and flux uncertainties (and their correlations), while measurements of the $C P$-violating phase $\left(\delta_{C P}\right)$ additionally require an accurate understanding of the asymmetry in neutrino and antineutrino cross sections, and in muon and electron neutrino cross sections. The reported significant reduction in the total cross section and flux uncertainties, and the demonstrated ability of ND280 Upgrade to set powerful constraints from neutrino and antineutrino data independently, therefore suggests a correspondingly large constraint on the pertinent model systematic uncertainty on measurements of $\theta_{23}$ and $\delta_{C P}$. In particular it can be noted that the (anti) neutrino total cross section uncertainty is reduced to below the $\sim(2.9 \%) 2.5 \%$ expected statistical uncertainty on the electron neutrino samples at Hyper-K [39]. Although the asymmetry in muon and electron neutrino cross sections is not directly constrained, its root cause can at least partially stem from a mismodeling of the details of the nuclear ground state [40,41] which has been shown to be well measured, although it can be noted that another significant component of this uncertainty can stem from the modelling of radiative corrections to cross sections [42], which is not considered in this work.

The sensitivity studies presented here are based on the simulation of the detector performances expected with ND280 Upgrade and includes a relatively sophisticated, but still incomplete, set of nuclear uncertainties. Conservative assumptions have been taken when possible, e.g., considering mostly the kinematics projected in the transverse plane to minimize the dependence on flux modeling, leaving parameters unconstrained, and fitting the neutrino and antineutrino samples separately, thus not including the correlation between neutrino and antineutrino cross-section and flux uncertainties. Overall the results demonstrate the interesting potential model constraints enabled by exploiting proton and neutron kinematics measured at the near detector for future oscillation analyses using ND280 Upgrade.

While it is shown that most parameter constraints plateau at an acceptable level for Hyper-K era statistics, some show scope for improvement even beyond this limit. This is particularly true for those relating to the flux constraint from the hydrogen enhanced region of $\delta p_{T}$. At very high statistics improved (and more robust) sensitivities may also be gained by using finer binning or adding additional dimensions to the fit. This could be realized by either additional beam exposure for ND280 Upgrade and/or future further upgrades to increase the target mass, for example by using the methods suggested in Refs. [43,44].

\section{ACKNOWLEDGMENTS}

This work was initiated in the framework of the T2K Near Detector upgrade project, fruitful discussions in this context with our colleagues are gratefully acknowledged. This project has received funding from the European Union's Horizon 2020 research and innovation programme under the Marie Skłodowska-Curie grant agreement No. 754496. It also received support from the Russian Science Foundation Grant No. 19-12-00325 and the support in the framework of the State project "Science" by the Ministry of Science and Higher Education of the Russian Federation under the Contract No. 075-15-2020778. This work was supported by P2IO LabEx (No. ANR10-LABX-0038-Project "BSMNu") in the framework "Investissements d'Avenir" (No. ANR-11-IDEX-000301) managed by the Agence Nationale de la Recherche (ANR), France, in addition to Agence Nationale de la Recherche (ANR), France, Grant No. ANR-19-CE31-0001 - project "SUNCORE". We acknowledge the support of Grant No. CNRS/IN2P3 and CEA. This work was also supported by the DOE, USA.

\section{APPENDIX A: FITTING WITH RECONSTRUCTED NUCLEON MOMENTUM}

As discussed in Sec. II B 1, the reconstructed initial state nucleon momentum $\left(p_{N}\right)$ [32] can be used as an alternative to the transverse momentum imbalance $\left(\delta p_{T}\right)$ [31] as an input variable in the fit. The former contains more information than that latter, incorporating an inferred longitudinal imbalance, in addition to the transverse imbalance, and so better sensitivity is in principle expected. However, this better sensitivity accompanies the potential need for additional systematic uncertainties. For example, while the shape of $\delta p_{T}$ is largely independent from neutrino energy and nucleonlevel physics [31], this will not be the case for $p_{N}$. We therefore choose the more conservative and simple approach of quoting the primary sensitivities using a fit in $\delta p_{T}$ but discuss here what would be obtained using $p_{N}$ instead.

Generally it is found that using $p_{N}$ in place of $\delta p_{T}$ has a relatively modest impact on the extracted sensitivities shown in Sec. III, although a notable improvement was found for some parameters. These improvements are shown in Fig. 13, demonstrating how $p_{N}$ offers better ability to distinguish npnh, $1 \mathrm{p} 1 \mathrm{~h}$ and pion production processes compared to $\delta p_{T}$. This behavior has previously been noted in e.g., Ref. [33]. 

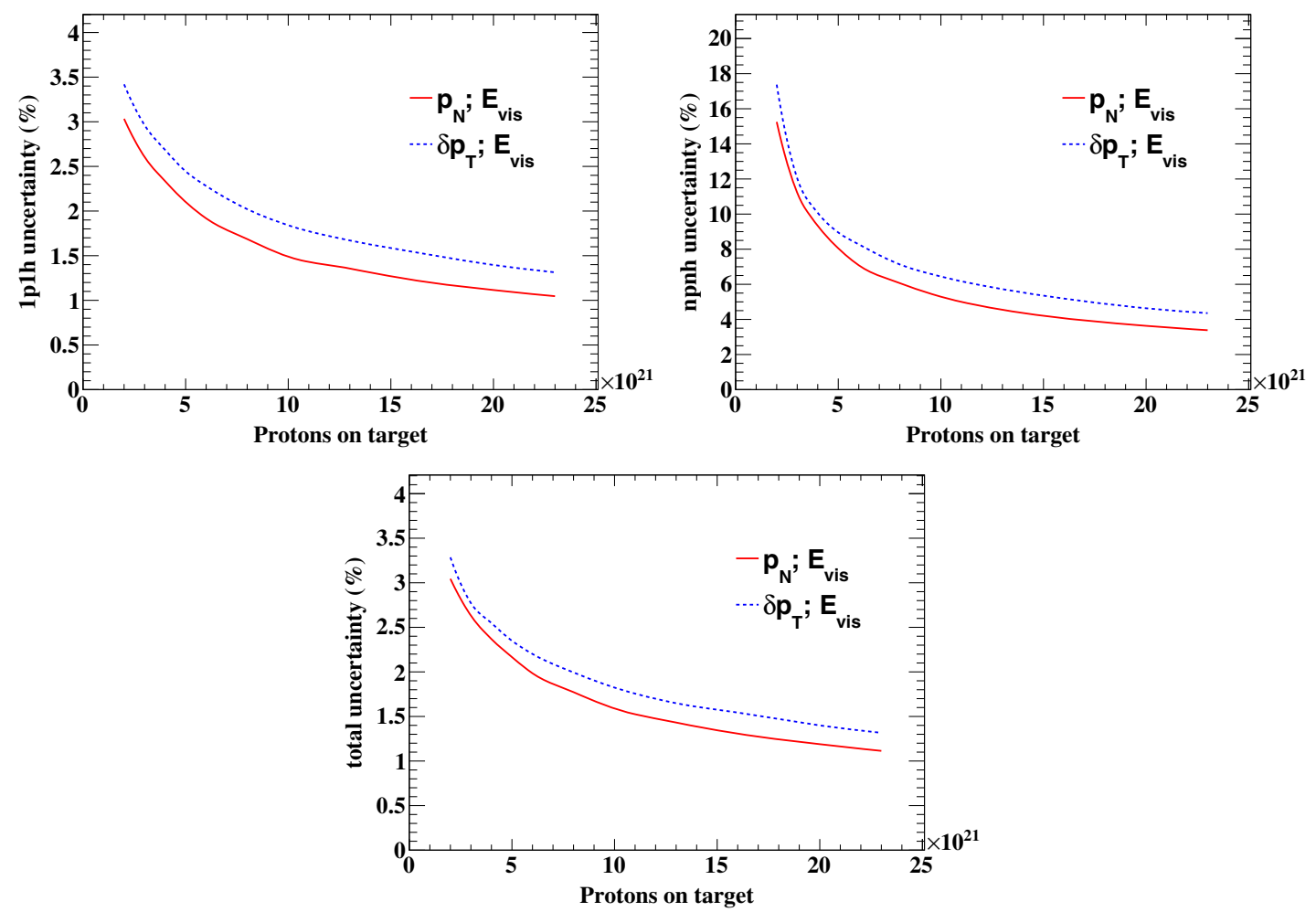

FIG. 13. The $1 \sigma$ sensitivity to the $1 \mathrm{p} 1 \mathrm{~h}$ (upper), npnh (middle) and total (lower) cross-section normalizations as a function of POT for neutrino interactions when fitting the reconstructed $\mathrm{CC} 0 \pi$ data binned in $E_{\mathrm{vis}}$ and either $\delta p_{T}$ or $p_{N}$.

\section{APPENDIX B: HISTOGRAMS INPUT TO THE FIT}

The full set of input histograms used in the fit projected onto single dimensions for neutrino and antineutrino interactions are shown in Fig. 14. The binning matches the one used in the fits. The histograms are shown in reconstructed kinematic quantities following the $\mathrm{CC} 0 \pi$ event selection and are broken down by interaction mode.
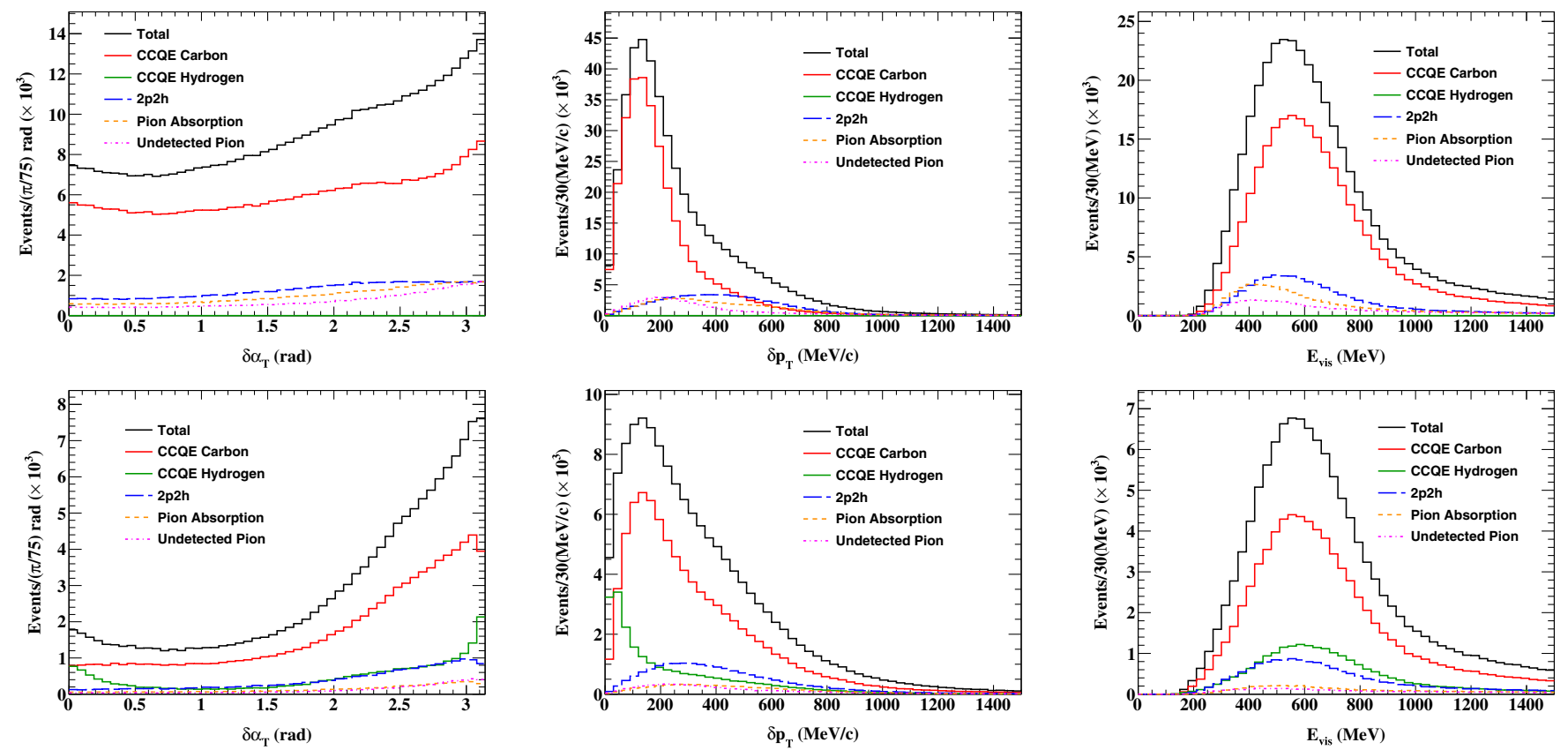

FIG. 14. One dimensional projections of the two-dimensional input histograms used to determine the sensitivities, shown here for $1 \times 10^{22}$ POT for neutrino (top) and antineutrino (bottom) interactions. The binning shown is that which is used in the fits. 
[1] L. Alvarez-Ruso et al., Prog. Part. Nucl. Phys. 100, 1 (2018).

[2] K. Abe et al. (T2K Collaboration), Nucl. Instrum. Methods Phys. Res., Sect. A 659, 106 (2011).

[3] K. Abe et al. (Hyper-Kamiokande Collaboration), arXiv: 1805.04163.

[4] K. Abe et al. (T2K Collaboration), arXiv:1901.03750.

[5] P. A. Amaudruz et al. (T2K ND280 FGD Collaboration), Nucl. Instrum. Methods Phys. Res., Sect. A 696, 1 (2012).

[6] N. Abgrall et al. (T2K ND280 TPC Collaboration), Nucl. Instrum. Methods Phys. Res., Sect. A 637, 25 (2011).

[7] D. Attié et al., Nucl. Instrum. Methods Phys. Res., Sect. A 957, 163286 (2020).

[8] A. Blondel et al., J. Instrum. 13, P02006 (2018).

[9] A. Korzenev et al., J. Phys. Soc. Jpn. Conf. Proc. 27, 011005 (2019).

[10] K. Abe et al. (T2K Collaboration), Nucl. Instrum. Methods Phys. Res., Sect. A 659, 106 (2011).

[11] K. Abe et al. (T2K Collaboration), Nature (London) 580, 339 (2020); Nature (London) 583, E16 (2020).

[12] K. Abe et al. (T2K Collaboration), Phys. Rev. D 103, 112008 (2021).

[13] L. Munteanu, S. Suvorov, S. Dolan, D. Sgalaberna, S. Bolognesi, S. Manly, G. Yang, C. Giganti, K. Iwamoto, and C. Jesús-Valls, Phys. Rev. D 101, 092003 (2020).

[14] A. Blondel et al., J. Instrum. 15, P12003 (2020).

[15] D. Attié et al., Nucl. Instrum. Methods Phys. Res., Sect. A 1025, 166109 (2022).

[16] Y. Hayato, Acta Phys. Pol. B 40, 2477 (2009).

[17] Y. Hayato and L. Pickering, Eur. Phys. J. Spec. Top. (2021), 10.1140/epjs/s11734-021-00287-7.

[18] K. Abe et al. (T2K Collaboration), Phys. Rev. D 87, 012001 (2013); Phys. Rev. D 87, 019902(A) (2013).

[19] http://t2k-experiment.org/wp-content/uploads/T2Kflux2016 .tar, accessed: 2019.

[20] O. Benhar, A. Fabrocini, S. Fantoni, and I. Sick, Nucl. Phys. A579, 493 (1994).

[21] J. Nieves, I. Ruiz Simo, and M. J. Vicente Vacas, Phys. Rev. C 83, 045501 (2011).

[22] D. Rein and L. M. Sehgal, Phys. Lett. 104B, 394 (1981); Phys. Lett. 106B, 513(E) (1981).
[23] M. Glück, E. Reya, and A. Vogt, Eur. Phys. J. C 5, 461 (1998).

[24] A. Bodek and U. K. Yang, AIP Conf. Proc. 792, 257 (2005).

[25] T. Sjostrand, Comput. Phys. Commun. 82, 74 (1994).

[26] L. Aliaga et al. (NuSTEC Collaboration), in NuSTEC Workshop on Neutrino-Nucleus Pion Production in the Resonance Region (2020), https://arxiv.org/abs/2011 .07166.

[27] E. S. P. Guerra et al., Phys. Rev. D 99, 052007 (2019).

[28] P. Stowell et al., J. Instrum. 12, P01016 (2017).

[29] K. Abe et al. (T2K Collaboration), Phys. Rev. D 98, 032003 (2018).

[30] X. G. Lu et al. (MINERvA Collaboration), Phys. Rev. Lett. 121, 022504 (2018).

[31] X. G. Lu, L. Pickering, S. Dolan, G. Barr, D. Coplowe, Y. Uchida, D. Wark, M. O. Wascko, A. Weber, and T. Yuan, Phys. Rev. C 94, 015503 (2016).

[32] A. P. Furmanski and J. T. Sobczyk, Phys. Rev. C 95, 065501 (2017).

[33] S. Dolan, arXiv:1810.06043.

[34] E. J. Moniz, I. Sick, R. R. Whitney, J. R. Ficenec, R. D. Kephart, and W. P. Trower, Phys. Rev. Lett. 26, 445 (1971).

[35] A. Bodek and T. Cai, Eur. Phys. J. C 79, 293 (2019).

[36] K. Niewczas and J. T. Sobczyk, Phys. Rev. C 100, 015505 (2019).

[37] S. Dytman, Y. Hayato, R. Raboanary, J. Sobczyk, J. T. Vidal, and N. Vololoniaina, Phys. Rev. D 104, 053006 (2021).

[38] R. J. Barlow and C. Beeston, Comput. Phys. Commun. 77, 219 (1993).

[39] K. Abe et al. (Hyper-Kamiokande Collaboration), arXiv: 1805.04163.

[40] A. Nikolakopoulos, N. Jachowicz, N. Van Dessel, K. Niewczas, R. González-Jiménez, J. M. Udías, and V. Pandey, Phys. Rev. Lett. 123, 052501 (2019).

[41] A. M. Ankowski, Phys. Rev. C 96, 035501 (2017).

[42] O. Tomalak, Q. Chen, R. J. Hill, and K. S. McFarland, arXiv:2105.07939.

[43] S. Berns, A. Boyarintsev, S. Hugon, U. Kose, D. Sgalaberna, A. De Roeck, A. Lebedynskiy, T. Sibilieva, and P. Zhmurin, J. Instrum. 15, P10019 (2020).

[44] A. Boyarintsev et al., J. Instrum. 16, P12010 (2021). 